\title{
A Fundamental Solution for a Biharmonic Finite-Difference Operator ${ }^{1}$
}

\author{
By R. Bruce Simpson
}

1. Introduction. Several authors have described a fundamental solution for the five-point finite-difference operator which approximates the Laplacian differential operator in the plane, and its asymptotic relation to a fundamental solution of the Laplacian has been known for some time [3], [7]. Recently an explicit bound for the difference between these two functions has been given by Mangad [6]. In a paper, [5], in which he estimates the difference between the Green's functions of the Dirichlet problem over a rectangle for the Laplace differential operator, and a Laplace difference operator, Laasonen shows how such estimates may be used to derive convergence rates for finite-difference approximations to Poisson's equation under very mild restrictions on the inhomogeneous term. It is the object of this paper to establish similar estimates to those of Mangad's for fundamental solutions of the biharmonic differential and difference operators which will enable analyses similar to those of Laasonen's to be made for biharmonic boundary value problems [8]. We consider any bounded region of the plane, and a square grid of mesh size $h$ covering the region. We construct a fundamental solution for the biharmonic operator in the region, and by an analogous procedure, we construct a discrete fundamental solution, defined at the grid points in the region, for the thirteen-point finite-difference operator which approximates the biharmonic operator with truncation error of order 2 [4]. The constructions are made so as to enable us to estimate the difference between these two functions as the mesh spacing varies.

By first extending slightly the estimate of Mangad to give a bound for the difference between the first divided differences of the continuous and discrete fundamental solutions to the corresponding Laplacian operators, we can obtain a similar estimate for the convergence of the first differences of the discrete biharmonic fundamental solution to the differences of the continuous one. The manner of extending these results to certain polyharmonic difference operators will be apparent from the constructions used here.

2. Preliminaries. Points of the plane, the set $E_{2}$, will be denoted by vectors $x$, with coordinates in a rectangular coordinate system $\left(x_{1}, x_{2}\right)$, and the length of $x$ will be given by $|x|=\left(x_{1}{ }^{2}+x_{2}{ }^{2}\right)^{1 / 2}$. We shall indicate the mesh points of a square grid of mesh size $h$ covering the plane and such that the coordinate axes are grid lines by $E_{h}$, and the points of $E_{h}$ will be denoted by vectors, $P$, written as capital letters. For a region $D$ of the plane, we define a corresponding set of grid points by $D_{h} \equiv \bar{D} \cap E_{h} . C_{\epsilon}(x)$ is to be the open disc of radius $\epsilon$ centered on $x$, and $S_{a}(x)$ is the square of side length $a$, oriented as parallel to the grid squares, and centered

Received August 10, 1966.

1 This work was supported by the National Science Foundation Grant NSF-GP-3666 and was part of the author's Ph.D. thesis at the University of Maryland. 
on $x$. For a function $V\left(P_{1}, P_{2}\right)$, defined on $E_{h}$, we introduce the first divided differences

$$
\delta_{h P_{1}} V\left(P_{1}, P_{2}\right) \equiv \frac{V\left(P_{1}+h, P_{2}\right)-V\left(P_{1}, P_{2}\right)}{h}
$$

and

$$
\bar{\delta}_{h P_{2}} V\left(P_{1}, P_{2}\right) \equiv \frac{V\left(P_{1}, P_{2}\right)-V\left(P_{1}, P_{2}-h\right)}{h}
$$

and the discrete Laplacian difference operator

$$
\Delta_{h} V(P)=\sum_{i=1}^{2} \bar{\delta}_{h P_{i}} \delta_{h P_{i}} V(P) .
$$

The set $N_{1}(P) \equiv\left\{Q\left|Q \in E_{h},\right| P-Q \mid \leq h\right\}$ is the set of arguments at which $V(Q)$ is required to form $\Delta_{h} V(P)$. For a set of grid points $D_{h}, N_{1}\left(D_{h}\right) \equiv\left\{Q \mid Q \in E_{h}\right.$, $Q \in N_{1}(P)$ for some $\left.P \in D_{h}\right\}$. The thirteen-point discrete biharmonic difference operator [4], denoted by $\Delta_{h}{ }^{2}$, can be defined by adopting a property of its continuous counterpart, $\Delta^{2}$, i.e.

$$
\Delta_{h}^{2} V(P) \equiv \Delta_{h}\left(\Delta_{h} V(P)\right) ;
$$

evidently $N_{2}(P) \equiv N_{1}\left(N_{1}(P)\right)$ is the set of arguments at which $V(Q)$ is required to form $\Delta_{h}^{2} V(P)$.

While the following device for estimating certain discrete sums has been used by Bramble and Hubbard, [2], there appears to be no explicit reference for it.

LEMмA 1. Let $f(x)$ be a nonnegative function, integrable over a region $R^{\prime}$ in $E_{2}$, and subharmonic in a subregion $R$. Then

$$
h^{2} \sum_{T \in D_{h}} f(T) \leqq \frac{4}{\pi} \int_{R^{\prime}} f d A
$$

for any region $D$ such that $T \in D_{h}, C_{h / 2}(T) \subseteq R \subseteq R^{\prime}$.

Proof. The proof is an application of the solid mean-value inequality for subharmonic functions, which in two dimensions is

$$
f(P) \leqq \frac{1}{\pi r^{2}} \int_{C_{r}(P)} f d A .
$$

In particular, taking $r=h / 2$ and $P \in D_{h}$

$$
f(P) \leqq \frac{4}{\pi h^{2}} \int_{C_{h / 2}(P)} f d A \leqq \frac{4}{\pi h^{2}} \int_{S_{h}(P)} f d A ;
$$

multiplying (3) by $h^{2}$, and summing over $D_{h}$ proves the result. We will use multiindices $\alpha=\left(\alpha_{1}, \alpha_{2}\right)$ with $|\alpha|=\alpha_{1}+\alpha_{2}$ and the symbol $D^{\alpha} f=\partial^{|\alpha|} f(x) / \partial x_{1}{ }^{\alpha_{1}} \partial x_{2}{ }^{\alpha_{2}}$ to describe derivatives.

Lemma 2. Let $f(x)$ be harmonic in a region $R$, so that $f(x)=\operatorname{Re} F(z)$, where $F(z)$ $i$ an analytic function of $z=x_{1}+i x_{2}$ in $R$. Then

$$
\left|D^{\alpha} f\right| \leqq\left|\frac{d^{|\alpha|} F(z)}{d z^{|\alpha|}}\right| \text { in } R
$$


Proof. First let us consider the case when $\alpha=\left(\alpha_{1}, 0\right)$. Then, since the partial derivatives are taken along the real axis,

$$
\frac{\partial^{\alpha_{1}} \operatorname{Re} F(z)}{\partial x_{1}^{\alpha_{1}}}=\operatorname{Re} \frac{d^{\alpha_{1}} F(z)}{d z^{\alpha_{1}}}
$$

so that

$$
\left|D^{\alpha} f\right|=\left|\operatorname{Re} \frac{d^{\alpha_{1}} F(z)}{d z^{\alpha_{1}}}\right| \leqq\left|\frac{d^{\alpha_{1}} F(z)}{d z^{\alpha_{1}}}\right| .
$$

Now let $g(x)=\operatorname{Im} F(z)=\operatorname{Re}(-i F(z))$ in $R$. Then, for $\alpha_{2}$ odd, $\alpha_{2}=2 m-1$,

$$
\frac{\partial^{\alpha_{2}} f}{\partial x_{2}^{\alpha_{2}}}=(-1)^{m} \frac{\partial^{\alpha_{2}} g}{\partial x_{1}^{\alpha_{2}}}
$$

and

$$
\left|\frac{\partial^{|\alpha|} f}{\partial x_{1}{ }^{\alpha_{1}} \partial x_{2}{ }^{\alpha_{2}}}\right|=\left|\frac{\partial^{|\alpha|} g}{\partial x_{1}{ }^{|\alpha|}}\right| \leqq\left|\frac{d^{|\alpha|}-i F(z)}{d z^{|\alpha|}}\right| \leqq\left|\frac{d^{|\alpha|} F(z)}{d z^{|\alpha|}}\right| .
$$

On the other hand, for $\alpha_{2}$ even, $\alpha_{2}=2 m$,

$$
\frac{\partial^{\alpha_{2}} f}{\partial x_{2}^{\alpha_{2}}}=(-1)^{m} \frac{\partial^{\alpha_{2}} f}{\partial x_{1}^{\alpha_{2}}}
$$

and, again,

$$
\left|\frac{\partial^{|\alpha|} f}{\partial x_{1}^{\alpha_{1}} \partial x_{2}{ }^{\alpha_{2}}}\right|=\left|\frac{\partial^{|\alpha|} f}{\partial x_{1}{ }^{|\alpha|}}\right| \leqq\left|\frac{d^{|\alpha|} F(z)}{d z^{\mid \alpha !}}\right| .
$$

From this lemma, we conclude directly that for $x \neq s$, there is a constant $k(\alpha)$, depending only on $|\alpha|$, such that

$$
\left|D_{x}^{\alpha} \log \right| x-s|| \leqq \frac{k(\alpha)}{|x-s|^{|\alpha|}} ;
$$

In deriving the estimate of this paper, we shall use $k$ and $K$ to denote generic constants which are independent of $h$ (their appearance in successive inequalities does not represent the same value, but only the fact that there is some constant for which the inequality is valid).

Let $A(P ; Q)=A\left(P_{1}, P_{2} ; Q_{1}, Q_{2}\right)$ be a function of two grid points $P$ and $Q$, and let $L_{h}$ stand for either $\Delta_{h}$ or $\Delta_{h}{ }^{2}$. Then $A(P ; Q)$ is defined to be a fundamental solution for $L_{h}$ on the set of grid points $D_{h} \subset E_{h}$ if it is defined on $N^{2}\left(D_{h}\right) \times N_{2}\left(D_{h}\right)$, and satisfies both

$$
\begin{aligned}
& L_{h P} A(P ; Q)=\frac{\delta(P ; Q)}{h^{2}} ; \quad P \in D_{h} ; Q \in D_{h} . \\
& L_{h Q} A(P ; Q)=\frac{\delta(P ; Q)}{h^{2}},
\end{aligned}
$$

Here $\delta(P ; Q)$ denotes the Kronecker delta symbol, and the subscript on the op- 
erator indicates that the operator acts on $A(P ; Q)$ as a function of that variable which depends parametrically on the other.

It is shown by McCrea and Whipple [7], that the function defined for $m$ and $n$ integers

$$
g(m, n) \equiv \frac{1}{2 \pi} \int_{0}^{\pi} \frac{1-\exp (-|m| y) \cos n s}{\sinh y} d s
$$

where $y$ lies on the branch of the root of $\cos s+\cosh y=2$ which varies between 0 and $\left|\cosh ^{-1} 3\right|$ could be used to define a fundamental solution, $\Gamma_{h}(P ; Q)$, for $-\Delta_{h}$ in $E_{h}$

$$
\Gamma_{h}(P ; Q) \equiv g\left(\frac{P_{1}-Q_{1}}{h}, \frac{P_{2}-Q_{2}}{h}\right)-\frac{3}{4 \pi} \log 2-C_{e}
$$

where $C_{e}$ is Euler's constant. If we denote the radially symmetric fundamental solution of the Laplacian $(-\Delta)$ by

$$
\gamma(x ; \xi)=\frac{1}{2 \pi} \log |x-\xi|
$$

then Mangad has shown [6] that

$$
\left|\gamma(P ; Q)-\Gamma_{h}(P ; Q)\right| \leqq 54(h /|P-Q|)^{2} .
$$

Consider a bounded subset $D$ of $E_{2}$, and a square $\Sigma$ the sides of which are grid lines and such that $\bar{D}$ lies in the interior of $\Sigma$; and let $G_{h}(P ; Q)$ be defined on $\Sigma_{h} \times \Sigma_{h}$, for any $Q \in \Sigma_{h}$, by

$$
\begin{aligned}
-\Delta_{h P} G_{h}(P ; Q) & =\frac{\delta(P ; Q)}{h^{2}}, \quad P \in \Sigma_{h}-(\partial \Sigma)_{h}, \\
G_{h}(P ; Q) & =0, \quad P \in(\partial \Sigma)_{h}
\end{aligned}
$$

(i.e., $G_{h}(P ; Q)$ is the discrete Green's function for $\left.\Sigma_{h}\right)$. From (7), it can be seen that we can choose positive constants $k_{0}$ and $k_{1}$, depending on the distance $d$ from $\partial \Sigma$ to $\bar{D}$, such that for $Q \in D_{h}, h<d / 3, P \in(\partial \Sigma)_{h}$

$$
\begin{aligned}
& H_{0}(P ; Q) \equiv-G_{h}(P ; Q)-k_{0}+\Gamma_{h}(P ; Q) \leqq 0, \\
& H_{1}(P ; Q) \equiv-G_{h}(P ; Q)+k_{1}+\Gamma_{h}(P ; Q) \geqq 0 .
\end{aligned}
$$

Since $\Delta_{h} H_{i}(P ; Q)=0, i=0,1$, for $P \in \Sigma_{h}-(\partial \Sigma)_{h}$, by the discrete maximumminimum principle [1],

$$
G_{h}(P ; Q)-k_{1} \leqq \Gamma_{h}(P ; Q) \leqq G_{h}(P ; Q)+k_{0}
$$

i.e.,

$$
\left|\Gamma_{h}(P ; Q)\right| \leqq G_{h}(P ; Q)+k_{2}, \quad k_{2}=\max \left(k_{0}, k_{1}\right) .
$$

Laasonen [5] has shown that for $a \equiv$ side length of $\Sigma$

$$
G_{h}(P ; Q) \leqq G_{h}(Q ; Q) \leqq .354(\log (a / h+1))
$$

which allows us to state

LEMmA 3. For a given $h_{0}$ and bounded subset $D$ of $E_{2}$, there is a constant $k_{2}$ depend- 
ing on $h_{0}$ and $D$ only, such that for $h<h_{0}$,

$$
\max _{P, Q \in D_{h}}\left|\Gamma_{h}(P ; Q)\right| \leqq .354|\log h|+k_{2} .
$$

The technique of Mangad, [6], in obtaining (7), can easily be modified to prove the following

Theorem 1. There exists a numerical constant $c$ such that for $|P-Q|>h$,

$$
\left|\delta_{h} \Gamma_{h}(P ; Q)-\delta_{h} \gamma(P ; Q)\right| \leqq c h^{2} /|P-Q|^{3}
$$

where $\delta_{h}$ denotes any first difference with respect to either $P_{i}$ or $Q_{i}, i=1,2$.

We shall indicate the necessary modifications of Mangad's proof of (7), and we observe that while our outline is simplified to show only the existence of $c$, a more lengthy calculation could be performed in a similar manner to provide $c$ explicitly. Since

$$
\begin{aligned}
\delta_{h Q_{i}} \Gamma_{h}(P ; Q) & =-\bar{\delta}_{h P_{i}} \Gamma_{h}(P ; Q), \\
\delta_{h Q_{i}} \gamma(P ; Q) & =-\bar{\delta}_{h P_{i}} \gamma(P ; Q),
\end{aligned}
$$

we may consider only differences with respect to $P$. Furthermore, from (5), and the symmetries of $g(m, n)$ [7] it can be seen that it is sufficient to consider $Q=$ $(0,0) \equiv 0$, and $P$ to be in the sector of the plane characterized by

$$
0 \leqq P_{2} \leqq P_{1}, \quad P \neq(0,0) \text { or }(h, 0) .
$$

However, it appears to be necessary to consider the $P_{1}$ and $P_{2}$ differences separately. From a standard Laplace transform, for $P_{i}=m_{i} h$,

$$
\delta_{h P_{2}} \gamma(P ; 0)=\frac{1}{2 \pi h} \int_{0}^{\infty}\left(\frac{\cos m_{2} s-\cos \left(m_{2}+1\right) s}{s}\right) e^{-m_{1} s} d s .
$$

Hence, with (4), we have, for $\cos s+\cosh y=2$

$$
\begin{aligned}
2 \pi\left|\delta_{h P_{2}}\left(\Gamma_{h}-\gamma\right)(P ; 0)\right|=\frac{1}{h} \mid & \int_{0}^{\pi}\left(\frac{\cos m_{2} s-\cos \left(m_{2}+1\right) s}{\sinh y}\right) e^{-m_{1} y} d s \\
& -\int_{0}^{\infty}\left(\cos m_{2} s-\cos \left(m_{2}+1\right) s\right) \frac{e^{-m_{1} s}}{s} d s \mid .
\end{aligned}
$$

(Here, and in the proofs to follow, we use the common notation $(f \pm g)(x)$ for the function $f(x) \pm g(x)$.)

We break up the right side of (11) as

$$
2 \pi\left|\delta_{h P_{2}}\left(\Gamma_{h}-\gamma\right)(P ; 0)\right| \leqq|A|+|B|+|C|,
$$

where

$$
\begin{aligned}
& A=\frac{1}{h} \int_{\epsilon}^{\infty}\left(\frac{\cos m_{2} s-\cos \left(m_{2}+1\right) s}{s}\right) e^{-m_{1} s} d s, \\
& B=\frac{1}{h} \int_{\epsilon}^{\pi}\left(\frac{\cos m_{2} s-\cos \left(m_{2}+1\right) s}{\sinh y}\right) e^{-m_{1} y} d s, \\
& C=\frac{1}{h} \int_{0}^{\epsilon}\left(\cos m_{2} s-\cos \left(m_{2}+1\right) s\right)\left(\frac{e^{-m_{1} y}}{\sinh y}-\frac{e^{-m_{1} s}}{s}\right) d s,
\end{aligned}
$$


for some choice of $\epsilon$ in $0<\epsilon<1$, independent of $h$. The estimation of terms $|A|$ and $|B|$ of (12) is entirely analogous to that in [6]; we indicate how $|C|$, the more complicated term, is estimated. From Lemma 2 of [6], for $0 \leqq s \leqq 1, s-s^{3} / 10$ $\leqq y \leqq s$, and a straightforward calculation shows that $\lim _{s \rightarrow 0+}(\sinh y) / s=\left(\frac{2}{3}\right)^{1 / 2}$. Hence for some $\epsilon>0$

$$
\frac{e^{-m_{1} y}}{\sinh y} \geqq \frac{e^{-m_{1} s}}{s} \text { when } 0<s \leqq \epsilon
$$

and we have

$$
\begin{aligned}
|C| & \leqq \frac{1}{h} \int_{0}^{\epsilon} s\left|\frac{e^{-m_{1} y}}{\sinh y}-\frac{e^{-m_{1} s}}{s}\right| d s \\
& \leqq \frac{1}{h} \int_{0}^{\epsilon}\left(\frac{\exp m_{1} \frac{s^{3}}{10}}{1-\frac{s^{2}}{10}}-1\right) e^{-m_{1} s} d s \\
& \leqq \frac{10}{\left(10-\epsilon^{2}\right) h} \int_{0}^{\epsilon}\left(\exp \left(\frac{m_{1} s^{3}}{10}\right)-1+\frac{s^{2}}{10}\right) e^{-m_{1} s} d s \\
& \leqq \frac{1}{\left(10-\epsilon^{2}\right) h}\left(\int_{0}^{\epsilon} m_{1} s^{3} \exp \left(-m_{1}\left(s-\frac{s^{3}}{10}\right)\right) d s+\int_{0}^{\epsilon} s^{2} e^{-m_{1} s} d s\right) .
\end{aligned}
$$

Now on $(0, \epsilon) s-s^{3} / 10 \geqq\left(1-\epsilon^{2} / 10\right) s$, so that

$$
\exp \left(-m_{1}\left(s-s^{3} / 10\right)\right) \leqq \exp \left(-\left(1-\epsilon^{2} / 10\right) m_{1} s\right)
$$

and

$$
\begin{aligned}
|C| & \leqq \frac{m_{1}}{\left(10-\epsilon^{2}\right) h} \int_{0}^{\infty} s^{3} \exp \left(-\left(1-\frac{\epsilon^{2}}{10}\right) m_{1} s\right) d s+\frac{h^{2}}{\left(10-\epsilon^{2}\right) m_{1}{ }^{3} h^{3}} \\
& \leqq \frac{m_{1}}{\left(10-\epsilon^{2}\right) h}\left(\frac{1}{\left(1-\epsilon^{2} / 10\right) m_{1}}\right)^{4}+k(\epsilon) \frac{h^{2}}{|P|^{3}} \leqq k(\epsilon) \frac{h^{2}}{|P|^{3}}
\end{aligned}
$$

since from (10), $P_{1}=m_{1} h \geqq|P| / 2^{1 / 2}$. We note that the more complicated procedure of [6] would avoid the uncertainty about the range of $\epsilon$ introduced at (13) and would permit explicit estimation of $c$.

Similarly, we have

$$
2 \pi\left|\delta_{h P_{1}}\left(\Gamma_{h}-\gamma\right)\left(I^{\prime} ; 0\right)\right| \leqq\left|A^{\prime}\right|+\left|B^{\prime}\right|+\left|C^{\prime}\right|
$$

for

$$
\begin{aligned}
& A^{\prime}=\frac{1}{h} \int_{\epsilon}^{\infty} \frac{\cos m_{2} s}{s}\left(e^{-m_{1} s}-e^{-\left(m_{1}+1\right) s}\right) d s \\
& B^{\prime}=\frac{1}{h} \int_{\epsilon}^{\pi} \frac{\left(1-e^{-y}\right) e^{-m_{1} y}}{\sinh y} \cos m_{2} s d s \\
& C^{\prime}=\frac{1}{h} \int_{0}^{\epsilon} \cos m_{2} s\left(\frac{1-e^{-y}}{\sinh y} e^{-m_{1} y}-\frac{1-e^{-s}}{s} e^{-m_{1} s}\right) d s
\end{aligned}
$$

and, again, we will only indicate the treatment of $C^{\prime}$. If $I$ is used to denote the 
integrand of $C^{\prime}$, we let $S^{+}\left(S^{-}\right)$be the subset of $(0, \epsilon)$ on which $I$ is positive (negative). Since $\sinh y \geqq y \geqq s-s^{3} / 10, s \geqq y$ for $\epsilon<1$ [6, Lemma 2], on $S^{+}$

$$
\begin{aligned}
|I| & \leqq\left(\frac{1-e^{-s}}{s-s^{3} / 10}\right) e^{-m_{1} y}-\left(\frac{1-e^{-s}}{s}\right) e^{-m_{1} s} \\
& \leqq \frac{1-e^{-s}}{s}\left(\frac{\exp \left(-m_{1}\left(s-\frac{s^{3}}{10}\right)\right)}{1-s^{2} / 10}-e^{-m_{1} s}\right) \\
& \leqq \frac{k}{1-\epsilon^{2} / 10} e^{-m_{1} s}\left(\exp \left(\frac{m_{1} s^{3}}{10}\right)-1-\frac{s^{2}}{10}\right)
\end{aligned}
$$

Hence

$$
\frac{1}{h} \int_{S^{+}}|I| d s \leqq \frac{k}{h} \int_{0}^{\epsilon}\left(\exp \left(\frac{m_{1} s^{3}}{10}\right)-1+\frac{s^{2}}{10}\right) e^{-m_{1} s} d s
$$

which was estimated in (14). On $S^{-}$

$$
|I| \leqq \frac{1-e^{-s}}{s} e^{-m_{1} s}-\frac{1-e^{-y}}{\sinh y} e^{-m_{1} s}
$$

now $\sinh y \leqq \sinh s \leqq s+\left(s^{3} / 3 !\right) \cosh \epsilon$ and $1 / \sinh y \geqq\left(1-b s^{2}\right) / s$ where $b=$ [cosh $\epsilon] / 3$ !. Thus on $S^{-}$

$$
\begin{aligned}
& |I| \leqq\left\{\frac{1-e^{-s}}{s}-\left(1-\exp \left(-s+\frac{s^{3}}{10}\right)\right)\left(\frac{1-b s^{2}}{s}\right)\right\} e^{-m_{1} s}
\end{aligned}
$$

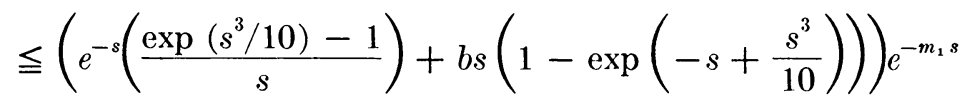

$$
\begin{aligned}
& \leqq\left(\frac{s^{2}}{10}+b s^{2}\right) \exp \left(-s+\frac{s^{3}}{10}\right) e^{-m_{1} s} \leqq k s^{2} e^{-m_{1} s}
\end{aligned}
$$

for $0 \leqq s \leqq \epsilon<1$, and

$$
\frac{1}{h} \int_{S^{-}}|I| d s \leqq \frac{1}{h} \int_{0}^{\infty} k s^{2} e^{-m_{1} s} d s .
$$

However, the right-hand side of (15) was also estimated at (14). These, then, with the corresponding estimates for $|A|,|B|,\left|A^{\prime}\right|$, and $\left|B^{\prime}\right|$ and the symmetries of $\Gamma_{h}$, and $\gamma$ conclude the estimate.

3. A Fundamental Solution for the Biharmonic Differential Operator. To define a function on $E_{2} \times E_{2}$ which is a fundamental solution for $\Delta^{2}$ in a bounded region $D$, let $L$ be a circle centered on the origin and containing $D$, and let $L_{0}$ be a circle with the same center but with radius $r_{0}$ equal to twice the radius of $L . L_{1}$ is to be a larger circle than $L_{0}$, centered on the origin, with radius $r_{1}$. Let $f(s) \in C_{0}{ }^{\infty}(I)$, where $I$ denotes the real line, $0 \leqq f(s) \leqq 1$, and $f(s) \equiv 1$ for $0 \leqq s \leqq r_{0}, f(s) \equiv 0$ for $s \geqq r_{1}$; we define $\eta(x)$ on $E_{2}$ by $\eta(x)=f(|x|)$ and we define $B(x ; \xi)$ on $E_{2} \times E_{2}$ as

$$
B(x ; \xi) \equiv \int \eta(t) \gamma(x ; t) \gamma(\xi ; t) d A_{t}
$$


For convenience in the sequel, we will assume that $r_{1}=2 r_{0}$, and we summarize some of the properties of this function in the following theorem.

THeorem 2. (i) $B(x ; \xi)$ is a fundamental solution for $\Delta^{2}$ in $D$.

(ii) $B(x ; \xi) \in C^{1}\left(E_{2} \times E_{2}\right)$ and a modulus of continuity for any of its first derivatives is $\omega(\delta)=K \delta|\log \delta|$.

(iii) For $x \neq \xi, x, \xi \in L_{0}$,

$$
\Delta_{x} B(x ; \xi)=\Delta_{\xi} B(x ; \xi)=\gamma(x ; \xi) .
$$

(iv) For $x \neq \xi, B(x ; \xi)$ is an infinitely differentiable function of $x$ and $\xi$, and its derivatives are continuous in the sense of a function of four variables when $x$ and $\xi$ vary in disjoint subsets of $L_{0}$.

(v) There is a constant $K$, depending on $r_{0}$ and $\alpha$, such that for $x \neq \xi x, \xi \in$ $L_{0},|\alpha| \geqq 2$,

$$
\left|D_{x}^{\alpha} B(x ; \xi)\right| \leqq \frac{K(|\log | x-\xi||+1)}{|x-\xi|^{|\alpha|-2}} .
$$

Proof. (i) To see that

$$
\int B(x ; \xi) \Delta^{2} \phi(\xi) d A_{\xi}=\phi(x)
$$

for all $\phi(x) \in C_{0}^{\infty}(D)$, we need only justify the interchange of order of integration in $\iint^{t} \eta(t) \gamma(x ; t) \gamma(\xi ; t) \Delta^{2} \phi(\xi) d A_{l} d A_{\xi}$ and observe that (a) $\Delta^{2} \phi(\xi)=-\Delta(-\Delta \phi(\xi))$, (b) $\gamma(\xi ; t)=\gamma(t ; \xi)$ is a fundamental solution for $-\Delta$, (c) $\eta(t) \equiv 1$ on the support of $\phi$.

(ii) Since

$$
\left|\int_{C_{\epsilon}(x)} \eta(t) \frac{1}{|x-t|} \frac{\partial|x-t|}{\partial x_{i}}\right| \log |\xi-t|\left|d A_{t}\right|
$$

converges to zero as $\epsilon$ tends to zero uniformly for $(x, \xi) \in L_{0} \times L_{0}$, we conclude that

$$
\frac{\partial B(x ; \xi)}{\partial x_{i}}=\int \eta(t) \frac{\partial \gamma(x ; t)}{\partial x_{i}} \gamma(\xi ; t) d A_{t}
$$

exists on $L_{0} \times L_{0}$. Suppose that

$$
\left|(x, \xi)-\left(x^{\prime}, \xi^{\prime}\right)\right|=\left(\sum_{i=1}^{2}\left(x_{i}-x_{i}{ }^{\prime}\right)^{2}+\left(\xi_{i}-\xi_{i}{ }^{\prime}\right)^{2}\right)^{1 / 2}<\delta,
$$

then $x^{\prime} \in C_{\delta}(x), \xi^{\prime} \in C_{\delta}(\xi)$ and

$$
\left|\frac{\partial B\left(x^{\prime} ; \xi^{\prime}\right)}{\partial x_{i}}-\frac{\partial B(x ; \xi)}{\partial x_{i}}\right| \leqq\left|I_{1}\right|+\left|I_{2}\right|+\left|I_{3}\right|
$$

where, setting $S=E_{2}-C_{2 \delta}(x)-C_{2 \delta}(\xi)$

$$
I_{1}=\int_{S} \eta(t)\left(\frac{\partial \gamma\left(t ; x^{\prime}\right)}{\partial x_{i}} \gamma\left(t ; \xi^{\prime}\right)-\frac{\partial \gamma(t ; x)}{\partial x_{i}} \gamma(t ; \xi)\right) d A_{t}
$$

and $I_{2}$ and $I_{3}$ are integrals having the same integrand, but taken over $C_{2 \delta}(x)$ and 
$C_{2 \delta}(\xi)$ respectively. Using Lemma 2 , for $(y, w) \in C_{\delta}(x) \times C_{\delta}(\xi)$

$$
\begin{aligned}
\mid \int_{S} \eta(t) & \frac{\partial \gamma(y ; t)}{\partial t_{i}} \frac{\partial \gamma(w ; t)}{\partial t_{j}} d A_{t} \mid \\
& \leqq\left(\int_{S} \eta(t)\left(\frac{k}{|y-t|}\right)^{2} d A_{t}\right)^{1 / 2}\left(\int_{S} \eta(t)\left(\frac{k}{|w-t|}\right)^{2} d A_{t}\right)^{1 / 2} \\
& \leqq k \int_{\delta}^{\lambda} \frac{d r}{r} \leqq k(\log \lambda-\log \delta)
\end{aligned}
$$

for $\lambda=r_{0}+r_{1}$. We can estimate $I_{1}$ as follows

$$
\left|I_{1}\right| \leqq|A|+|B|,
$$

where, setting $g(t)=\gamma(\xi ; t)+\gamma\left(\xi^{\prime} ; t\right) / 2$, and $s x+(1-s) x^{\prime}=x(s) \in C_{\delta}(x)$ for $0 \leqq s \leqq 1$,

$$
\begin{aligned}
A= & \int_{S} \eta(t) g(t) \frac{\partial}{\partial t_{i}}\left(\gamma(x ; t)-\gamma\left(x^{\prime} ; t\right)\right) d A_{t} \\
= & \sum_{k=1}^{2}\left(x_{k}-x_{k}^{\prime}\right) \int_{S} \eta(t) g(t) \int_{0}^{1}-\frac{\partial^{2} \gamma(x(s) ; t)}{\partial t_{k} \partial t_{i}} d s d A_{t} \\
= & \sum_{k=1}^{2}\left(x_{k}-x_{k}^{\prime}\right) \int_{0}^{1}\left(\int_{S} \frac{\partial \eta(t) g(t)}{\partial t_{i}} \frac{\partial \gamma(x(s) ; t)}{\partial t_{k}} d A_{t}\right. \\
& \left.\quad+\oint_{\partial S} n_{i} \eta(t) g(t) \frac{\partial \gamma(x(s) ; t)}{\partial t_{k}} d \sigma_{t}\right) d s,
\end{aligned}
$$

$\left(n_{1}, n_{2}\right)$ being the outer normal to $\partial S$. Now, using (21), we can estimate

$$
\int_{S} \frac{\partial \eta(t) g(t)}{\partial t_{i}} \frac{\partial \gamma(x(s) ; t)}{\partial t_{k}} d A_{t}
$$

uniformly for $s \in[0,1]$, and

$$
\begin{aligned}
& \left|\oint_{\partial S} n_{i}\left(\frac{\gamma(\xi ; t)+\gamma\left(\xi^{\prime} ; t\right)}{2}\right) \frac{\partial \gamma(x(s) ; t)}{\partial t_{k}} d \sigma_{t}\right| \\
& \quad \leqq \frac{k}{\delta}(\log \lambda-\log \delta) \oint_{\partial S} d \sigma_{t} \leqq K(\log \lambda-\log \delta) .
\end{aligned}
$$

Since $\left|x_{k}-x_{k}{ }^{\prime}\right|<\delta$, then, $|A| \leqq K \delta(\log \lambda-\log \delta)$. The estimation of $B$ is similar but simpler; i.e. for $\xi(s)=s \xi+(1-s) \xi^{\prime} \in C_{\delta}(\xi)$

$$
\begin{aligned}
B & =\int_{S} \frac{\eta(t)}{2}\left(\gamma(\xi ; t)-\gamma\left(\xi^{\prime} ; t\right)\right) \frac{\partial}{\partial t_{i}}\left(\gamma(x ; t)+\gamma\left(x^{\prime} ; t\right)\right) d A_{t} \\
& =\sum_{k=1}^{2}\left(\frac{\xi_{k}-\xi_{k}{ }^{\prime}}{2}\right) \int_{0}^{1} \int_{S} \eta(t)-\frac{\partial \gamma(\xi(s) ; t)}{\partial t_{k}} \frac{\partial}{\partial t_{i}}\left(\gamma(x ; t)+\gamma\left(x^{\prime} ; t\right)\right) d A_{t} d s .
\end{aligned}
$$

The volume integral is estimated using (21) and we have $|B| \leqq K \delta(\log \lambda-\log \delta)$ so that, for $\delta<\delta_{0}$,

$$
\left|I_{1}\right| \leqq K \delta|\log \delta|
$$

To complete our estimate for (10), we must consider $I_{2}$ and $I_{3}$; however, letting 
$J$ be either $C_{2 \delta}(x)$ or $C_{2 \delta}(\xi)$, it can be verified that, for $x^{\prime} \in C_{\delta}(x), \xi^{\prime} \in C_{\hat{\delta}}^{\prime}(\xi)$

$$
\left|\int_{J} \frac{\partial \gamma\left(x^{\prime} ; t\right)}{\partial x_{i}} \gamma\left(\xi^{\prime} ; t\right) d A_{t}\right| \leqq K \delta|\log \delta|
$$

for a uniform constant $K$, using Hölder's inequality. Using (24) to estimate $\left|I_{2}\right|$ and $\left|I_{3}\right|$, we have, from (19), that for $\delta<\delta_{0}$, there is a $K$, dependent on $\delta_{0}$ and $\lambda$ only, such that for $\left|(x, \xi)-\left(x^{\prime}, \xi^{\prime}\right)\right|<\delta$

$$
\left|\frac{\partial B\left(x^{\prime} ; \xi^{\prime}\right)}{\partial x_{i}}-\frac{\partial B(x ; \xi)}{\partial x_{i}}\right|<K \delta|\log \delta|
$$

which proves (ii).

(iii), (iv) Let $\Lambda_{1}$ and $\Lambda_{2}$ be two compact disjoint subsets of $E_{2}$, we wish to show that $D_{x}{ }^{\alpha} B(x ; \xi)$ is continuous on $\Lambda_{1} \times \Lambda_{2}$ for every $\alpha$. Let $4 \epsilon=\left|\Lambda_{1}-\Lambda_{2}\right| \equiv$ $\inf _{x} \in \Lambda_{\Lambda_{1} ; y} \in \Lambda_{\Lambda_{2}}|x-y|$ and take $\Omega$ to be a piecewise smooth, compact curve enclosing $\Lambda_{1}$ such that $\left|\Omega-\Lambda_{1}\right| \geqq \epsilon, i=1,2$. Let $Z$ be the interior of $\Omega$, and we assume for convenience that $Z \subset L_{0}$, i.e. $\eta(t) \equiv 1, t \in Z$. From (18), it can be seen that

$$
\begin{aligned}
\frac{\partial B(x ; \xi)}{\partial x_{i}}= & \int_{E_{2}-Z} \eta(t) \frac{\partial \gamma(x ; t)}{\partial x_{i}} \gamma(\xi ; t) d A_{t} \\
& +\int_{Z} \gamma(x ; t) \frac{\partial \gamma(\xi ; t)}{\partial t_{i}} d A_{t}+\oint_{\Omega}-n_{i} \gamma(x ; t) \gamma(\xi ; t) d \sigma_{t}
\end{aligned}
$$

where $\left(n_{1}, n_{2}\right)$ is the outer normal to $\Omega$. For $x \in \Lambda_{1}$ and $\xi \in \Lambda_{2}$, if the right side of (26) is differentiated under the integral signs with respect to $x_{j}$, the resulting integrals converge uniformly with respect to $x \in \Lambda_{1}$, i.e., using Gauss's theorem

$$
\begin{aligned}
\frac{\partial^{2} B(x ; \xi)}{\partial x_{i} \partial x_{j}}= & \int_{E_{2}-Z} \eta(t) \frac{\partial^{2} \gamma(x ; t)}{\partial x_{i} \partial x_{j}} \gamma(\xi ; t) d A_{t} \\
& +\int_{Z} \gamma(x ; t) \frac{\partial^{2} \gamma(\xi ; t)}{\partial t_{i} \partial t_{j}} d A_{t} \\
& +\oint_{\Omega} n_{i} \frac{\partial \gamma(x ; t)}{\partial t_{j}} \gamma(t ; \xi)-n_{j} \frac{\partial \gamma(\xi ; t)}{\partial t_{i}} \gamma(x ; t) d \sigma_{t} .
\end{aligned}
$$

In particular, if we choose $x \neq \xi, \Lambda_{1}=x, \Lambda_{2}=\xi, \Omega=C_{\epsilon}(x)$, (27) shows that

$$
\begin{aligned}
\Delta_{x} B(x ; \xi)= & \int_{E_{2}-C_{\epsilon}(x)} \eta(t) \Delta_{x} \gamma(x ; t) \gamma(\xi ; t) d A_{t} \\
& +\int_{C_{\epsilon}(x)} \gamma(x ; t) \Delta_{t} \gamma(\xi ; t) d A_{t} \\
& +\oint_{|x-t|=\epsilon}\left(\frac{\partial \gamma(x ; t)}{\partial n_{t}} \gamma(\xi ; t)-\frac{\partial \gamma(\xi ; t)}{\partial n_{t}} \gamma(x ; t)\right) d \sigma_{t} \\
= & \frac{1}{2 \pi \epsilon} \oint_{|x-t|=\epsilon} \gamma(\xi ; t) d \sigma_{t}-\frac{\log \epsilon}{2 \pi} \oint_{|x-t|=\epsilon} \frac{\partial \gamma(\xi ; t)}{\partial n_{t}} d \sigma_{t}=\gamma(\xi ; x)
\end{aligned}
$$

since $\gamma(x ; t)$ is harmonic for $x \neq t$, enabling us to employ the mean value theorem for harmonic functions for the first line integral above and to conclude that the second vanishes, proving (iii). 
Returning to the case of arbitrary but disjoint $\Lambda_{1}$ and $\Lambda_{2}$, we can continue in the manner in which (27) was obtained to see that for $x \in \Lambda_{1} \subset Z, \xi \in \Lambda_{2}$

$$
\begin{aligned}
D_{x}^{\alpha} B(x ; \xi)= & \int_{E_{2}-Z} \eta(t) \gamma(\xi ; t) D_{x}^{\alpha} \gamma(x ; t) d A_{t} \\
& +(-1)^{|\alpha|} \int_{Z} \gamma(x ; t) D_{t}^{\alpha} \gamma(\xi ; t) d A_{t} \\
& +\oint_{\Omega ; \mid} \sum_{\rho+\tau|=| \alpha !-1} n_{1} a_{\rho \tau} D_{t}{ }^{\rho} \gamma(x ; t) D_{t}{ }^{\tau} \gamma(\xi ; t) \\
& +n_{2} b_{\rho \tau} D_{t}{ }^{\rho} \gamma(x ; t) D_{t}{ }^{\tau} \gamma(\xi ; t) d \sigma_{t}
\end{aligned}
$$

where $a_{\rho \tau}, b_{\rho \tau}$ are numerical constants depending only on the multi-indices which are their subscripts. Since the integrand of the line integral in (28) is uniformly continuous for $(x, \xi, t) \in \Lambda_{1} \times \Lambda_{2} \times \Omega$, the line integral is continuous on $\Lambda_{1} \times \Lambda_{2}$. That the volume integrals define functions which are continuous on $\Lambda_{1} \times \Lambda_{2}$ can be seen from the approach taken in proving (ii).

(v) To obtain these estimates for the derivatives of $B(x ; \xi)$ when $x \neq \xi, x, \xi \in L_{0}$, we set in the proof of (iv) $x=\Lambda_{1}, \xi=\Lambda_{2}$ and $\Omega=C_{\epsilon}(x), \epsilon=|x-\xi| / 2$ and estimate the various terms on the right side of (28) using Lemma 2. E.g., setting $|\alpha|=a$,

$$
\begin{aligned}
\left|\int_{E_{2}-C_{\epsilon}(x)} \eta(t) D_{x}^{\alpha} \gamma(x ; t) \gamma(\xi ; t) d A_{t}\right| & \\
& \leqq k(\alpha)\left\{\int_{E_{2}-C_{\epsilon}(x)-C_{\epsilon}(\xi)} \eta(t) \frac{1}{|x-t|^{a}}|\log | \xi-t|| d A_{t}\right. \\
& \left.\quad+\int_{C \epsilon(\xi)} \frac{1}{|x-t|^{a}}|\log | \xi-t|| d A_{t}\right\} \\
& \leqq k(\alpha)\left\{\left(\left|\log \frac{|x-\xi|}{4}\right|+\log \lambda\right) \int_{|x-\xi| / 4}^{\lambda}+\frac{d r}{r^{a-1}}\right. \\
& \leqq K \frac{|\log | x-\xi||+1}{|x-\xi|^{a-2}}
\end{aligned}
$$

where $K$ depends on $\alpha$ and $\lambda$. Similarly

$$
\left|\int_{C_{\epsilon}(x)} \gamma(x ; t) D_{t}^{\alpha} \gamma(\xi ; t) d A_{t}\right| \leqq K \frac{|\log | x-\xi||+1}{|x-\xi|^{a-2}}
$$

and the line integrals can easily be shown to satisfy the same estimate, for some $K$ depending only on $\alpha$ and $\lambda$.

4. A Fundamental Solution for the Biharmonic Difference Operator. The definition of $B(x ; \xi)$ is immediately suggestive of the following construction. We define $\Gamma_{h}(P ; t)$ to be the extension a.e. of $\Gamma_{h}(P ; Q)$ to $E_{h} \times E_{2}$ as

$$
\Gamma_{h}(P ; t)=\Gamma_{h}(P ; Q), \quad t \in S_{h}(Q),
$$


and let $B_{h}(P ; Q)$ be defined on $E_{h} \times E_{h}$ by

$$
B_{h}(P ; Q)=\int \eta(t) \Gamma_{h}(P ; t) \Gamma_{h}(Q ; t) d A_{t}
$$

Theorem 3. $B_{h}(P ; Q)$ is a fundamental solution of $\Delta_{h}{ }^{2}$ in $L_{0 h}$.

Proof. This result follows immediately from applying $\Delta_{h P}^{2}$ to $B_{h}(P ; Q)$, observing (2) and the fact that $\Gamma_{h}(P ; S)$ is a fundamental solution for $-\Delta_{h}$.

The apparent fact that $B_{h}(P ; Q)$ is an approximation to $B(P ; Q)$ is given quantitative substance by the main results of this paper, Theorems 4 and 5 .

THeOREM 4 . For any constants $h_{0}$ and $l_{0}$ satisfying $2 r_{0}>l_{0}>6 h_{0}$, there exists a constant $M$ depending on $h_{0}, l_{0}$ and $r_{0}$, such that for $h<h_{0}$,

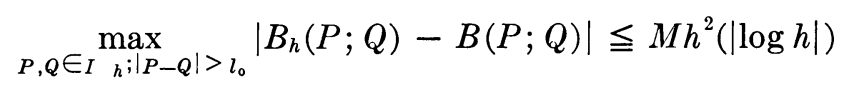

and a constant $M_{1}$ depending on $h_{0}$ and $r_{0}$ such that for $h<h_{0}$

$$
\max _{P, Q \in L_{0 h}}\left|B_{h}(P ; Q)-B(P ; Q)\right| \leqq M_{1} h^{2}(\log h)^{2}
$$

where $L_{0}$ is the circle of radius $r_{0}$ centered on the origin.

Proof. By definition, we have

$$
\begin{aligned}
B_{h}(P ; Q)-B(P ; Q)= & \int \eta(t)\left(\Gamma_{h}(P ; t) \Gamma_{h}(Q ; t)-\gamma(P ; t) \gamma(Q ; t)\right) d A_{t} \\
= & \int \eta(t)\left(\Gamma_{h}-\gamma\right)(P ; t)\left(\frac{\Gamma_{h}+\gamma}{2}\right)(Q ; t) d A_{t} \\
& +\int \eta(t)\left(\Gamma_{h}-\gamma\right)(Q ; t)\left(\frac{\Gamma_{h}+\gamma}{2}\right)(P ; t) d A_{t} \\
\equiv & I_{1}+I_{2}
\end{aligned}
$$

where $I_{1}$ and $I_{2}$ are defined to be the two integrals on the preceding line. Since they are similar in form, it is sufficient to show how $\left|I_{1}\right|$ can be estimated. We introduce a piecewise constant function

$$
\begin{aligned}
& \gamma_{h}(P ; t) \equiv \gamma(P ; T), \quad t \in S_{h}(T), T \notin N_{2}(P), \\
& \gamma_{h}(P ; t) \equiv \Gamma_{h}(P ; T), \quad t \in S_{h}(T), T \in N_{2}(P) .
\end{aligned}
$$

Then

$$
\begin{aligned}
I_{1}= & \int \eta(t)\left(\gamma-\gamma_{h}\right)(P ; t)\left(\frac{\gamma+\Gamma_{h}}{2}\right)(Q ; t) d A_{t} \\
& +\int \eta(t)\left(\gamma_{h}-\Gamma_{h}\right)(P ; t)\left(\frac{\gamma+\Gamma_{h}}{2}\right)(Q ; t) d A_{t} \\
\equiv & J_{1}+J_{2} .
\end{aligned}
$$

We wish to use the following observation in estimating $J_{1}$. Let $M_{i \phi}$ denote a uniform bound over $S_{h}(T)$ for the absolute value of the $i$ th derivatives of $\phi(t) \in C^{i}\left[S_{h}(T)\right]$ and consider $f(t) \in C^{2}\left[S_{h}(T)\right], g(t) \in C^{1}\left[S_{h}(T)\right]$. Then, using Taylor's expansions, it is immediate that 


$$
\frac{1}{h^{2}}\left|\int_{S_{h}(T)}(f(t)-f(T)) g(t) d A\right| \leqq \frac{h^{2}}{3}\left(M_{2 f}|g(T)|+M_{1 f} M_{1 \sigma}\right) .
$$

If $t \in S_{h}(T)$, and $T \notin N_{2}(P)$, then

$$
|P-t| \geqq(2 / 5)^{1 / 2}|P-T| ;
$$

hence, with Lemma 2,

$$
\begin{aligned}
& \max _{t \in S_{h}(T)}\left|\frac{\partial^{2} \gamma(P ; t)}{\partial t_{i} \partial t_{j}}\right| \leqq \frac{k}{|P-T|^{2}}, \\
& \max _{t \in S_{h}(T)}\left|\frac{\partial \gamma(P ; t)}{\partial t_{i}}\right| \leqq \frac{k}{|P-T|} .
\end{aligned}
$$

Noting that the restriction $l_{0}>6 h_{0}$ ensures that $N_{2}(Q) \cap N_{2}(P)$ is void, we have with $N_{2} \equiv N_{2}(P) \cup N_{2}(Q), f(t)=\gamma(P ; t), g(t)=\eta(t)\left(\left(\gamma+\Gamma_{h}\right) / 2\right)(Q ; t)$

$$
\begin{aligned}
\left|J_{1}\right| \leqq & h^{2} \sum_{T \in E_{E^{-}-N_{2}}} \frac{1}{h^{2}}\left|\int_{S_{h}(T)}(f(t)-f(T)) g(t) d A_{t}\right| \\
& +h^{2} \sum_{T \in N_{2}(P)} \frac{1}{h^{2}}\left(\int_{S_{h}(T)}|\gamma(P ; t)| d A_{t}+\int_{S_{h}(T)}\left|\Gamma_{h}(P ; t)\right| d A_{t}\right) \\
& \times\left(\max _{t \in S_{S^{h}}(T)}\left(|\gamma(Q ; t)|+\left|\Gamma_{h}(Q ; t)\right|\right)\right) \\
& +h^{2} \sum_{T \in N_{2}(Q)} \max _{t \in S_{h}(T)} \eta(t)|\gamma(P ; t)-\gamma(P ; T)| \\
& \times\left\{\frac{1}{h^{2}} \int_{S_{h}(T)}|\gamma(Q ; t)| d A_{t}+\left|\Gamma_{h}(Q ; T)\right|\right\} .
\end{aligned}
$$

From $\left|\Gamma_{h}(Q ; T)\right| \leqq|\gamma(Q ; T)|+54\left(h^{2} /|Q-T|^{2}\right)$, for $T \notin N_{2}(Q),|g(T)| \leqq$ $(1 / 2 \pi)|\log | Q-T||+6$ and, using (30)-(32), it can be seen that the first term on the right side of (33) is bounded by $S_{1}$, where

$$
\begin{aligned}
S_{1}= & k h^{2} \sum_{T \in L 1 h-N_{2}} h^{2}\left(\frac{1}{|P-T|^{2}}\left(\frac{1}{2 \pi}|\log | Q-T||+6\right)+\frac{1}{|P-T|} \frac{1}{|Q-T|}\right) \\
& +k h^{2} \sum_{r_{0} \leqq|T| \leqq r_{2}} h^{2}\left(\frac{1}{|P-T|}|\log | Q-T||\right) M_{1 \eta} .
\end{aligned}
$$

However, estimating the summand within and without $C_{l_{0} / 2}(Q)$, and using Lemma 1 ,

$$
\begin{aligned}
h^{2} \sum_{T \in L_{L_{1}} N_{2}} \frac{|\log | Q-T||}{|P-T|^{2}} \leqq & \left(\log 4 r_{0}-\log \left(\frac{l_{0}}{2}\right)\right) h^{2} \sum_{T \in \sum_{L_{1} h-N_{2}} \frac{1}{|P-T|^{2}}} \\
& +\frac{4}{l_{0}^{2}} h^{2}{ }_{|T-Q| \leqq l_{0} / 2 ; T \oplus_{N_{2}(Q)}}|\log | Q-T|| \\
\leqq & k\left(\log 4 r_{0}-\log h\right)+k_{1} .
\end{aligned}
$$

From Schwarz inequality, and Lemma 1

$$
h^{2} \sum_{T \in L_{1 h}{ }^{-N_{2}}} \frac{1}{|P-T|} \frac{1}{|Q-T|} \leqq k\left(\int_{h}^{4 r_{0}} \frac{d r}{r}\right) \leqq k\left(\log 4 r_{0}-\log h\right)
$$


and it is clear that the remaining terms of (35) can be estimated in a similar fashion, so that for any $h_{0}$, there is a constant $K$ depending on $h_{0}, l_{0}$ and $r_{0}$ such that

$$
S_{1} \leqq K h^{2}|\log h| \text { for } h<h_{0} \text {. }
$$

The remaining two terms on the right of (33) are each sums, multiplied by $h^{2}$, of 13 terms, where each term is bounded by $K|\log h|$ for $h<h_{0}$, for a suitable constant $K$ depending on $h_{0}, l_{0}$ and $r_{0}$. Hence, for a suitable constant $K$,

$$
\left|J_{1}\right| \leqq K h^{2}|\log h| \text { for } h<h_{0} \text {. }
$$

Using the estimate of $g(T)$ preceding (35), and also (6) and (7), and Lemma 3

$$
\begin{aligned}
\left|J_{2}\right| \leqq & \sum_{T \in E_{L^{-} N_{2}}}\left(54 \frac{h^{2}}{|P-T|^{2}}\right) \int_{S_{h}(T)} \eta(t)\left(\frac{|\log | Q-t||}{2 \pi}+6\right) d A_{t} \\
& +\sum_{T \in N_{2}(Q)} 54 \frac{h^{2}}{|P-T|^{2}} \int_{S_{h}(T)} \frac{\eta(t)}{2}\left(\frac{|\log | Q-t||}{2 \pi}+k_{2}-.354 \log h\right) d A_{t} \\
\leqq & K h^{2}|\log h| \text { for } h<h_{0}
\end{aligned}
$$

for a suitable constant $K$. This concludes the estimation of $I_{1}$ (Eq. (29)), but, as mentioned, $I_{2}$ is similar in form, hence (i) is established.

The second estimate (ii) is obtained by the same process, not using, however, $|P-Q| \geqq l_{0}$. If we examine the first term of (33), it is bounded by $S_{1}$ of (35) which can be estimated uniformly, as in (36) using

$$
\begin{aligned}
h^{2} \sum_{T \in L_{1} h^{-N_{2}}} & \frac{|\log | Q-T||}{|P-T|^{2}} \\
& \leqq\left(\log 4 r_{0}-\log h\right) h^{2} \sum_{T \in L_{1 h^{-} N_{2}}} \frac{1}{|P-T|^{2}} \leqq K(\log h)^{2},
\end{aligned}
$$

and the fact that (37) is already uniform in $P$ and $Q$. Hence, for $P$ and $Q$ in $L_{0 h}$, and $h<h_{0}$,

$$
S_{1} \leqq K h^{2}(\log h)^{2}
$$

for a constant $K$ depending on $r_{0}$ and $h_{0}$. The remaining two terms in (33) are sums, multiplied by $h^{2}$, of 13 terms, each of which is bounded, for $h<h_{0}$, by $K(\log h)^{2}$, for a suitable $K$ which depends only on $h_{0}$ and $r_{0}$. Hence, we have the analogous estimate to (38) uniform in $P, Q$

$$
\left|J_{1}\right| \leqq K h^{2}(\log h)^{2}
$$

for a suitable constant $K$. Similar modifications of (39) will show that

$$
\left|J_{2}\right| \leqq K h^{2}(\log h)^{2}
$$

for $P, Q \in L_{0 h}$ and $h<h_{0}$, which, with (41), establishes the second estimate.

We wish now to conclude our results by using Theorem 1 to establish an estimate similar to (i) of Theorem 4, for the first differences of the fundamental solutions of the biharmonic and discrete biharmonic operators.

Theorem 5. For $h_{0}<r_{0} / 5$, there exists a constant $M_{2}$, depending on $h_{0}$ and $r_{0}$, such that for any first-difference operator $\delta_{h}$, 


$$
\max _{P, Q \in L_{0 h} ;|P-Q|>5 h}\left|\delta_{h}\left(B(P ; Q)-B_{h}(P ; Q)\right)\right| \leqq \frac{M_{2} h^{2}|\log h|}{|P-Q|}
$$

for $h<h_{0}$.

Proof. While the idea of the proof is essentially the same as that of Theorem 4 , some alterations are necessary to provide the nonuniform estimate. For any two points $P, Q \in L_{0 h}$ such that $|P-Q|>5 h$, let $W$ be a smallest square containing $S_{h}(T)$, for any $T \in\left(C_{|P-Q|}(P) \cup C_{|P-Q|}(Q)\right)_{h},\left(C_{a}(b)\right.$ being the circle of radius $a$, centered on $b$ as defined above). Consider the grid lines running in a direction which make an angle of $45^{\circ}$ or greater with the line segment $P Q$, and choose a line running in this direction which is halfway between the grid lines of the considered direction and which is one of possibly two such lines that are nearest to the midpoint of $P Q$. (See Fig. 1.) This line will be labelled $J$, and coincides with a line of edges of squares $S_{h}(T)$ which comprise $W$. It divides $W$ into two rectangles, $W_{1}$ the rectangle con-

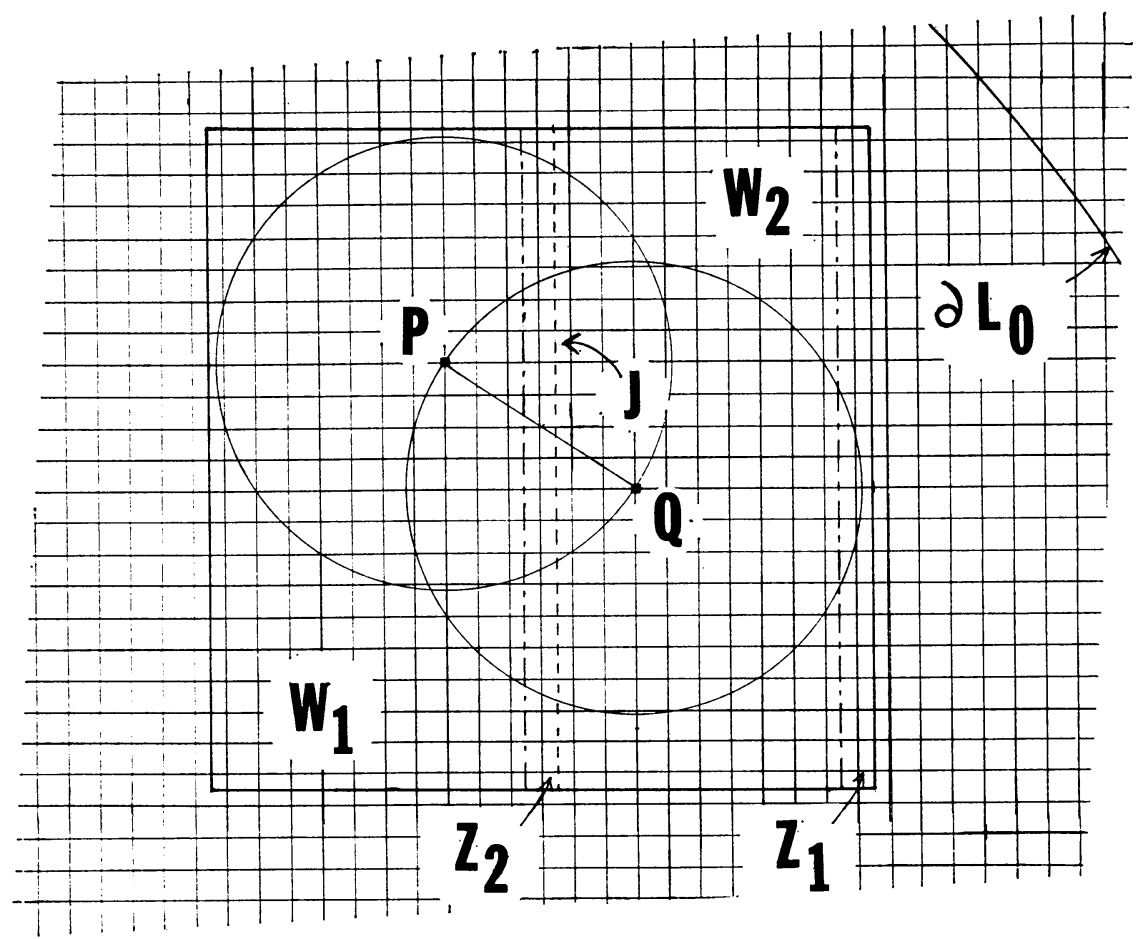

taining $P$ and $W_{2}$ the rectangle containing $Q$. Using this,

$$
\left|\delta_{h} B(P ; Q)-\delta_{h} B_{h}(P ; Q)\right| \leqq\left|I_{1}\right|+\left|I_{2}\right|+\left|I_{3}\right|
$$

where the $I_{i}$ are integrals with integrand

$$
\eta(t)\left(\delta_{h}\left(\Gamma_{h}(P ; t) \Gamma_{h}(Q ; t)\right)-\delta_{h}(\gamma(P ; t) \gamma(Q ; t))\right)
$$

taken over $t \in W_{1}, W_{2}$ and $E_{2}-W$, respectively. We proceed with the particular case $\delta_{h}=\delta_{h Q_{1}}$, the other cases being handled in exactly the same way. With

$$
G=\left\{T \mid T \in L_{1 h}, T \notin W\right\},
$$




$$
\begin{array}{rl}
\left|I_{3}\right| \leqq & \frac{h^{2}}{2} \sum_{T \in G}\left\{\left|\left(\Gamma_{h}+\gamma\right)(P ; T)\left(\delta_{h}\left(\Gamma_{h}-\gamma\right)(Q ; T)\right)\right|\right. \\
& +\left|\left(\Gamma_{h}-\gamma\right)(P ; T) \delta_{h}\left(\Gamma_{h}+\gamma\right)(Q ; T)\right| \\
& +2 \mid \gamma(P ; T) \delta_{h} \gamma(Q ; T) \frac{1}{h^{2}} \int_{S_{h}(T)} \eta(t) d A \\
5 & k h^{2}\left(h^{2} \sum_{T \in G} \frac{|\log | P-T||}{|Q-T|^{3}}+\frac{1}{|Q-T|} \frac{1}{|P-T|^{2}} \int_{S_{h}(T)} \gamma(P ; t) \delta_{h} \gamma(Q ; t) \eta(t) d A \mid\right\} \\
& \left.+\frac{1}{|Q-T|^{2}} \frac{1}{|P-T|}+M_{1 \eta}\left(\frac{1}{|P-T|} \frac{1}{|Q-T|}+\frac{|\log | P-T||}{|Q-T|^{2}}\right)\right)
\end{array}
$$

where we have used Theorem 1, (7) and (30). Using Lemma 1, and the techniques of the preceding proof, we have

$$
\left|I_{3}\right| \leqq k h^{2}|\log h| /|P-Q|
$$

Turning to the remaining terms, $I_{1}$ and $I_{2}$, we can use (9) to see that

$$
\begin{aligned}
I_{2}= & \int_{W_{2}}\left\{\Gamma_{h}(P ; t)\left(\Gamma_{h}\left(Q ; t_{1}-h, t_{2}\right)-\Gamma_{h}(Q ; t)\right) / h\right. \\
& \left.-\gamma(P ; t)\left(\gamma\left(Q ; t_{1}-h, t_{2}\right)-\gamma(Q ; t)\right) / h\right\} \eta(t) d A_{t} \\
= & \int_{W_{2}}\left\{\left(-\Gamma_{h}(P ; t) \Gamma_{h}(Q ; t)+\gamma(P ; t) \gamma(Q ; t)\right) / h\right\} \eta(t) d A_{t} \\
& +\int_{W_{3}}\left\{\left(\Gamma_{h}\left(P ; t_{1}+h, t_{2}\right) \Gamma_{h}(Q ; t)-\gamma\left(P ; t_{1}+h, t_{2}\right) \gamma(Q ; t)\right) / h\right\} \\
& \times \eta\left(t_{1}+h, t_{2}\right) d A_{t}
\end{aligned}
$$

where $W_{3}$ is the rectangle obtained by translating $W_{2}$ through a distance $h$ in the direction of the negative $t_{1}$ axis. We let $W_{4}=W_{2} \cap W_{3}$ and let $Z_{1}$ and $Z_{2}$ be the two rectangles $W_{2}-W_{4}$ and $W_{3}-W_{4}$. We observe that the $Z_{i}$ have width $h$ and length not exceeding $3|P-Q|+h$, and contain not more than $3|P-Q| / h+1$ points of $E_{h}$. To simplify the following expressions, we shall assume that $W \cup$ $W_{3} \subset L_{0}$, so that $\eta(t) \equiv 1$ on $W \cup W_{3}$. The modifications of the following estimates which are necessary if $W \cup W_{3}$ is not contained in $L_{0}$ can be seen from the procedures of the preceding proof or the last terms on the right sides of (45). From (47), it is apparent that

$$
\begin{aligned}
I_{2}= & \int_{W_{\star}}\left\{-\bar{\delta}_{h P_{1}} \Gamma_{h}(P ; t) \Gamma_{h}(Q ; t)+\bar{\delta}_{h P_{1}} \gamma(P ; t) \gamma(Q ; t)\right\} d A_{t} \\
& +\frac{1}{h} \int_{Z_{1}}\left\{\gamma(P ; t) \gamma(Q ; t)-\Gamma_{h}(P ; t) \Gamma_{h}(Q ; t)\right\} d A_{t} \\
& +\frac{1}{h} \int_{Z_{2}}\left\{\Gamma_{h}\left(P ; t_{1}+h, t_{2}\right) \Gamma_{h}(Q ; t)-\gamma\left(P ; t_{1}+h, t_{2}\right) \gamma(Q ; t)\right\} d A_{t} .
\end{aligned}
$$

The integral over $W_{4}$ in (48) and $I_{1}$ can both be treated in the following manner: 


$$
\begin{aligned}
\left|I_{1}\right| \leqq & h^{2} \sum_{T \in N_{2}(P)}\left\{\left|\Gamma_{h}(P ; T) \delta_{h} \Gamma_{h}(Q ; T)\right|+\frac{1}{h^{2}}\left|\int_{S_{h}(T)} \gamma(P ; t) \delta_{h} \gamma(Q ; t) d A_{t}\right|\right\} \\
& +\frac{h^{2}}{2} \sum_{T \in W_{h^{-}-N_{2}(P)}}\left\{\left|\left(\gamma+\Gamma_{h}\right)(P ; T) \delta_{h}\left(\Gamma_{h}-\gamma\right)(Q ; T)\right|\right. \\
& +\left|\left(\gamma-\Gamma_{h}\right)(P ; T) \delta_{h}\left(\Gamma_{h}+\gamma\right)(Q ; T)\right|+2 \mid \gamma(P ; T) \delta_{h} \gamma(Q ; T) \\
& \left.-\frac{1}{h^{2}} \int_{S_{h}(T)} \gamma(P ; t) \delta_{h} \gamma(Q ; t) d A_{t} \mid\right\}
\end{aligned}
$$

For $t \in W_{1},|Q-t| \geqq|P-Q| / 2(2)^{1 / 2},-h / 2$, i.e.

$$
\begin{aligned}
\frac{1}{|Q-t|} & \leqq \frac{2(2)^{1 / 2}}{|P-Q|}\left(1-\frac{(2)^{1 / 2} h}{|P-Q|}\right)^{-1} \\
& \leqq \frac{10(2)^{1 / 2}}{|P-Q|} \frac{1}{5-(2)^{1 / 2}}=\frac{k}{|P-Q|}
\end{aligned}
$$

since $|P-Q| \geq 5 h$. To estimate the sum of 13 terms which is the first sum on the right side of (49), we employ Lemma 3, Theorem 1 and (50)

$$
\begin{aligned}
h^{2} \sum_{T \in N_{2}(P)} & \left(\left|\Gamma_{h}(P ; T) \delta_{h} \Gamma_{h}(Q ; T)\right|+\frac{1}{h^{2}}\left|\int_{S_{h}(T)} \gamma(P ; t) \delta_{h} \gamma(Q ; t) d A_{t}\right|\right) \\
& \leqq 13\left\{k h^{2}(|\log h|+1)\left(\frac{1}{|P-Q|}+\frac{8 c h^{2}}{|P-Q|^{3}}\right)\right. \\
& \left.\quad+\frac{k}{|P-Q|} \int_{0}^{3 h} r(-\log r) d r\right\} \\
& \leqq k \frac{h^{2}|\log h|}{|P-Q|}
\end{aligned}
$$

for $h<h_{0}, k$ dependent on $h_{0}$. For $T \in W_{1 h}-N_{2}(P),\left|\left(\gamma+\Gamma_{h}\right)(P ; T)\right| \leqq$ $((1 / \pi)|\log | P-T||+12)$ and

$$
\left|\delta_{h}\left(\Gamma_{h}+\gamma\right)(Q ; T)\right| \leqq 2\left|\delta_{h} \gamma(Q ; T)\right|+8 c \frac{h^{2}}{|P-Q|^{3}} \leqq \frac{k}{|P-Q|}
$$

hence

$$
\begin{aligned}
& \frac{h^{2}}{2} \sum_{T \in W_{1 h}-N_{2}(P)}\left\{\left|\left(\gamma+\Gamma_{h}\right)(P ; T) \delta_{h}\left(\Gamma_{h}-\gamma\right)(Q ; T)\right|\right. \\
&\left.+\left|\left(\gamma-\Gamma_{h}\right)(P ; T) \delta_{h}\left(\Gamma_{h}+\gamma\right)(Q ; T)\right|\right\} \\
& \leqq \quad+\left(\frac { h ^ { 2 } } { 2 } \sum _ { T \in W _ { 1 h } - N _ { 2 } ( P ) } \left\{\left(\frac{1}{\pi}|\log | P-T||+12\right)\left(\frac{8 c h^{2}}{|Q-T|^{3}}\right)\right.\right. \\
& \\
&\left.\left.\leqq k \frac{h^{2}|\log h|}{|P-Q|}\right)^{2} \frac{k}{|P-Q|}\right\}
\end{aligned}
$$


Using (30) and Lemma 1 in the same manner as previously, it can be seen that the last term of (49) can be bounded by the last term in (51), hence

$$
\left|I_{1}\right| \leqq k \frac{h^{2}}{|P-Q|}|\log h|
$$

for a suitable constant $k$.

As the analysis of $I_{1}$ would provide the necessary estimate of the first integral in the expression (48) for $I_{2}$, we can complete the estimation of $I_{2}$ by considering the integrals over the strips $Z_{i}$; e.g. set

$$
\begin{aligned}
I_{4}= & \left|\frac{1}{h} \int_{Z_{1}} \gamma(P ; t) \gamma(Q ; t)-\Gamma_{h}(P ; t) \Gamma_{h}(Q ; t) d A_{t}\right| \leqq \frac{h}{2} \sum_{T \in Z_{1 h}}\left\{\mid\left(\gamma-\Gamma_{h}\right)(P ; T)\right. \\
& \left.\times\left(\gamma+\Gamma_{h}\right)(Q ; T)|+|\left(\gamma-\Gamma_{h}\right)(Q ; T)\left(\gamma+\Gamma_{h}\right)(P ; T) \mid\right\} \\
& +h \sum_{T \in Z_{1 h}}\left|\frac{1}{h^{2}} \int_{S_{h}(T)} \gamma(P ; t) \gamma(Q ; t) d A_{t}-\gamma(P ; T) \gamma(Q ; T)\right| .
\end{aligned}
$$

Using (7), we can see that the first sum on the right-hand side of (53) is bounded by

$$
\begin{aligned}
k h^{3} \sum_{T \in Z_{1 h}}\left\{\frac{1}{|P-T|^{2}}\left(2|\log | Q-T||+\frac{54}{25}\right)\right. & \\
& \left.+\frac{1}{|Q-T|^{2}}\left(2|\log | P-T||+\frac{54}{25}\right)\right\} .
\end{aligned}
$$

For $t \in S_{h}(T), T \in Z_{i}$, the triangle inequality gives $|P-t|+h /(2)^{1 / 2} \geqq|P-T|$, so that for a number $a$, obtained by the same calculation as gave (50), $1 /|P-t|$ $\leqq a /|P-T|$ and similarly $1 /|Q-t| \leqq a /|Q-T|$. Thus, from (30), it can be seen that the second sum on the right side of (53) is less than

$$
k h^{3} \sum_{T \in Z_{\perp_{h}}} \frac{|\log | P-T||}{|Q-T|^{2}}+\frac{2}{|P-T||Q-T|}+\frac{|\log | Q-T||}{|P-T|^{2}} .
$$

We observe that

$$
\frac{|P-Q|}{2}\left(\frac{1}{(2)^{1 / 2}}-\frac{1}{5}\right) \leqq \frac{|P-Q|}{2(2)^{1 / 2}}-\frac{h}{2} \leqq|P-T|
$$

and similarly $|Q-T| \geqq k|P-Q|$ when $T \in Z_{i}$, and using these inequalities and noting the remarks preceding (48) we see that (54) and (55) are bounded for some constant $k$ by $k h^{2}|\log h| /|P-Q|$; e.g.,

$$
h^{3} \sum_{T \in Z_{1}} \frac{|\log | P-T||}{|Q-T|^{2}} \leqq k \frac{h^{2}|\log | P-Q||}{|P-Q|^{2}} h \sum_{T \in Z_{1}} 1 \leqq k_{1} \frac{h^{2}|\log h|}{|P-Q|} .
$$

Since the integral over $Z_{2}$ in (48) can be treated in the same manner, we have, for some $k$

$$
\left|I_{2}\right| \leqq k \frac{h^{2}|\log h|}{|P-Q|}
$$

which with (46), (52) and (44) shows the estimate given in the theorem to be valid 
when $\delta_{h}=\delta_{h Q_{1}}$. As mentioned, however, the other cases are not essentially different and so we shall consider the result proven.

California Institute of Technology

Pasadena, California 91109

1. J. H. Bramble \& B. E. Hubbard, "On the formulation of finite difference analogues of the Dirichlet problem for Poisson's equation," Numer. Math., v. 4, 1962, pp. 313-327. MR 26 \#7157.

2. J. H. Bramble \& B. E. Hubbard, "A priori bounds on the discretization error in the numerical solution of the Dirichlet problem," Contributions to Differential Equations, Vol. 2, 1963, pp. 229-251. MR 26 \#7158.

3. R. J. DufFin \& D. H. Shaffer, "Asymptotic expansion of double Fourier transforms," Duke Math. J., v. 27, 1960, pp. 581-596. MR 22 \#280.

4. L. V. Kantorovich \& V. I. Krylov, Approximate Methods of Higher Analysis, Interscience, New York; Noordhoff, Groningen, 1958. MR 21 \#5268.

5. P. Laasonen, "On the solution of Poisson's difference equation," J. Assoc. Comput. Mach., v. 5, 1958, pp. 370-382. MR $22 \# 12726$.

6. M. MANGAD, "Bounds for the two-dimensional discrete harmonic Green's function," Math. Comp., v. 20, 1966, pp. 60-67.

7. W. H. McCrea \& F. J. W. Whipple, "Random paths in two and three dimensions," Proc. Roy. Soc. Edinburgh, v. 60, 1940, pp. 281-298. MR 2, 107

8. R. B. Simpson, Finite Difference Methods for T'wo Biharmonic Boundary Value Problems, Ph.I). Thesis, University of Maryland, 1966. 